\title{
Turismo rural comunitario: una alternativa para el desarrollo socioeconómico de la comunidad El Ostional, San Juan del Sur, Rivas, Nicaragua
}

\section{Rural community tourism: an alternative for socioeconomic development in the El Ostional community, San Juan del Sur, Rivas, Nicaragua}

\author{
Carmen Anielka Arróliga Montenegro1, Francisco J. Zamora Jarquín² \\ ${ }^{1}$ MSc. en Desarrollo Rural / ORCID: https://orcid.org/0000-0002-2621-6809 / carmen.arroliga@ci.una.edu.ni \\ 2 MSc. en Sistemas integrales de Producción Agropecuaria en el Trópico. Ex decano de la Facultad Desarrollo Rural (q.e.p.d) \\ Universidad Nacional Agraria, Facultad de Desarrollo Rural
}

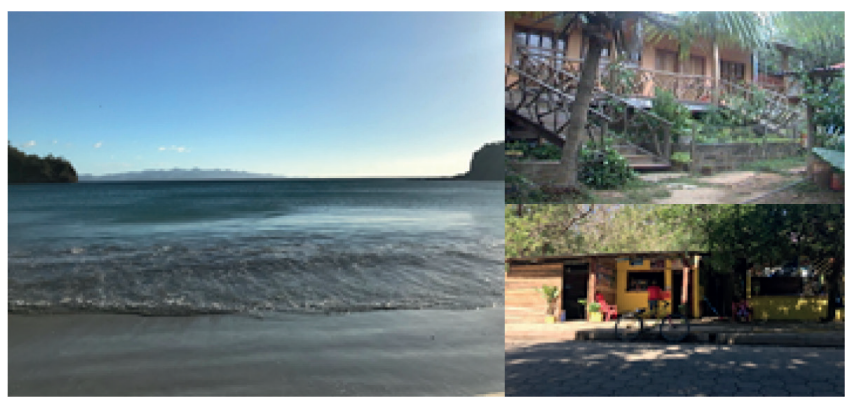

\section{RESUMEN}

El turismo rural comunitario es una alternativa social y económica al turismo tradicional. Este estudio pretende comprobar si el modelo de desarrollo turístico rural comunitario aplicado en la comunidad El Ostional se está gestionando de manera correcta y comprobar de esta forma si hay impacto en la mejora de la calidad de vida de la población, promoviendo el rescate de la identidad cultural. Para conocer estos aspectos es necesario describir los factores socioeconómicos como organización, equidad de género, y función de la mujer, capacitación, economía comunitaria y la vinculación del turismo con otras fuentes de ingreso y posteriormente evaluar su modelo turístico rural comunitario, para esto se aplicó la metodología de Vigilancia en Sitios Centinela utilizada por el Centro de Investigación de Enfermedades Tropicales que combina métodos cuantitativos y cualitativos, utilizando como unidad de análisis los hogares para las variables sociales y las fuentes de ingresos para variables económicas. El Turismo Rural Comunitario en El Ostional es cada vez más importante para el municipio de San Juan del Sur, tanto que, los proyectos del gobierno y de la entidad reguladora del turismo, tienen a la comunidad identificada como el principal atractivo para así descongestionar la afluencia de turistas a San Juan del Sur. El modelo turístico de El Ostional permite generar beneficios socioeconómicos directamente a la cooperativa de turismo e indirectamente al resto de los pobladores.

Palabras clave: desarrollo sostenible, neorruralidad, desarrollo rural, estrategia, dinamizador económico, modelo integrado.

\begin{abstract}
Community-based Rural Tourism is a social and economic alternative to traditional tourism. This study aims to verify if the model of community-based Rural Tourism development applied in the El Ostional community is being managed correctly and thus check if there is an impact on improving the quality of life of the population, promoting the rescue of cultural identity. To know these aspects it is necessary to describe the socioeconomic factors such as organization, gender equity and the role of women, training, community economy and the linkage of tourism with other sources of income and subsequently evaluate its rural community tourism model. For this purpose, the Sentinel Site Surveillance methodology used by the Tropical Disease Research Center was applied that combines quantitative and qualitative methods, using households as the unit of analysis for social variables and sources of income for economic variables. Community Rural Tourism in El Ostional is increasingly important for the municipality of San Juan del Sur, so much so that, the projects of the government and the tourism regulatory entity, have the community identified as the main attraction in order to decongest the influx of tourists to San Juan del Sur. The tourist model of El Ostional allows the generation of socioeconomic benefits directly to the tourism cooperative and indirectly to the rest of the inhabitants.
\end{abstract}

Keywords: Sustainable development, neo-rurality, rural development, strategy, economic dynamizer, integrated model.
Recibido: 21 de enero del 2020

Aceptado: 30 de octubre del 2020

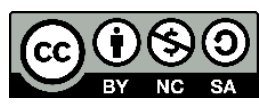

Los artículos de la revista La Calera de la Universidad Nacional Agraria, Nicaragua, se comparten bajo términos de la licencia Creative Commons: Reconocimiento, No Comercial, Compartir Igual. Las autorizaciones adicionales a las aquí delimitadas se pueden obtener en el correo edgardo.jimenez@ci.una.edu.ni

C Copyright 2020. Universidad Nacional Agraria (UNA). 


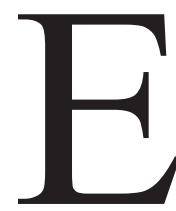

1 turismo ha venido creciendo de forma sostenida, colocándose como una de las principales actividades económicas generadoras de divisas y de beneficios al país. Según el Banco Central de Nicaragua (BCN, 2018), al primer semestre del año 2017, la actividad turística generó 392.2 millones de dólares, reflejando así un crecimiento del $29 \%$ en relación al 2016 .

El turismo rural representa una alternativa de desarrollo económico, social y cultural. Tiene grandes ventajas para una comunidad al ser una fuente generadora de empleos y de motivar a la población a conservar el medio ambiente, ya que el entorno natural es su sustento. En Nicaragua existen diferentes comunidades que están desarrollando modelos de turismo rural comunitario. Un ejemplo de esto es la comunidad El Ostional, municipio de San Juan del Sur, departamento de Rivas. Esta comunidad, según José de la Cruz, Presidente de la Cooperativa de Turismo de El Ostional, COOPETUR (comunicación personal, 4 de octubre 2017), está organizada como cooperativa de turismo desde el 2002 y ha trabajado con la mayoría de sus pobladores, gracias a su organización y a que ha logrado generar un cambio de mentalidad que insta a sus comunitarios a cuidar su entorno y a aprovecharlo responsablemente, lo que ha contribuido en una mejora de su calidad de vida, permitiendo que el turismo permanezca como una actividad económica complementaria.

En Nicaragua hay poca información sobre el impacto socioeconómico que genera el desarrollo turístico rural comunitario. Existen datos estadísticos de la actividad a nivel general, como, por ejemplo, la entrada de turistas al país y el dinero que generan, pero no existe información acerca de la actividad turística rural comunitaria como tal, que nos permita determinar si es una efectiva estrategia de desarrollo. Por ello la finalidad de este estudio es analizar el modelo de desarrollo turístico rural comunitario que implementa la comunidad El Ostional y comprobar si su gestión es sostenible en los aspectos sociales y cómo impacta en la dinámica económica.

\section{MATERIALES Y MÉTODOS}

Ubicación del área del estudio. La comunidad El Ostional se encuentra ubicada en el municipio de San Juan del Sur, departamento de Rivas a $159 \mathrm{~km}$ al sur de Managua, capital de Nicaragua entre los $11^{\circ} 6^{\prime} 33.22^{\prime \prime}$ y los $11^{\circ} 6^{\prime} 54.62^{\prime \prime}$ de latitud Norte y $85^{\circ} 45^{\prime} 36.62^{\prime \prime}$ y $85^{\circ} 45^{\prime} 55.46^{\prime \prime}$ de longitud Oeste.

La investigación se tipifica en mixta (cualitativacuantitativa), se basa en comprender y analizar el fenómeno en su estado, utilizando el método inductivo- deductivo. Según el diseño empleado, es de tipo no experimental debido a que se observó el fenómeno tal y en su contexto natural, también es una investigación explicativa, porque además de describir el fenómeno, se buscó la explicación del comportamiento de las variables.
Se utilizó la metodología de Vigilancia en Comunidades Centinelas, utilizada por el Centro de Investigación de Enfermedades Tropicales (CIET Internacional-Nicaragua) desde 1984, como un proceso complementario al sistema rutinario de información con posibilidades de ser utilizada en diversos países y con fines de vigilancia en distintos campos de acción.

Se trabajó la relación entre el crecimiento económico y el bienestar subjetivo que la gente percibe. Los indicadores subjetivos son un complemento esencial de los indicadores objetivos. Estos indicadores económicos básicos (objetivos y subjetivos) utilizados en el análisis del turismo, se concibieron como elementos que, a falta de estadísticas y control del turismo, permiten dimensionar la importancia económica del sector y de algunas de sus características más importantes, desde la perspectiva de la oferta. El estudio se desarrolló en tres etapas:

Etapa I. Preliminar o de organización del estudio. Se seleccionó la comunidad El Ostional, basándose en el conocimiento, experiencia y balance de opiniones de las comisiones de turismo y actores locales (Cámaras de Turismo, Alcaldías, Instituto Nicaragüense de Turismo y otras asociaciones). También en el nivel de relación económica con la actividad turística del destino (comunidades dedicadas a las actividades pesqueras, agrícolas y comerciales), la concentración poblacional y la accesibilidad del lugar.

Se identificaron las fuentes de información secundaria, lo que permitió definir el problema y el modelo turístico rural comunitario que se desarrolla en la comunidad. Los libros, páginas web, investigaciones científicas y otros documentos, fueron los insumos para la construcción del marco de referencia, algunos de ellos fueron suministrados por el Instituto Nicaragüense de Turismo (INTUR), la Alcaldía de San Juan del Sur y por el presidente de COOPETUR.

Etapa II. Central o de ejecución. En esta etapa se definieron las variables que corresponden a: modelo turístico, organización, equidad de género y función de la mujer, capacitación, economía comunitaria y la vinculación del turismo con otras fuentes de ingreso; y se construyeron instrumentos de registro de información como encuestas y guía de entrevista, las que previa a su aplicación fueron validadas.

Las encuestas fueron aplicadas en 61 hogares de un total de 81, debido a que el resto de las familias en los hogares no desearon participar en el estudio o porque sus habitantes se encontraban fuera de la comunidad.

Las entrevistas fueron dirigidas a informantes claves como al presidente de COOPETUR José de la Cruz Sánchez, al secretario político Julio Lacayo, al Responsable de Turismo de la Alcaldía de San Juan del Sur, Yader Collado 
y a la Encargada de Promoción de la Sub delegación INTUR de San Juan del Sur, Lidia Mendoza; todos estos informantes permitieron describir y analizar el comportamiento de la actividad turística en la comunidad en función de las variables definidas.

Etapa III. Procesamiento de datos. El ordenamiento y procesamiento de la información obtenida, se realizó mediante un procedimiento coherente, lógico y armónico, una vez recopilada la información, se ordenó, procesó y analizó.

\section{RESULTADOS Y DISCUSIÓN}

Modelo turístico. En la comunidad El Ostional se implementa un modelo turístico integrado, asociado generalmente a las pequeñas y medianas empresas; es un elemento coadyuvante que favorece el surgimiento y desarrollo del turismo en el que se da una vinculación de la experiencia turística con el ambiente y la población local. Con este modelo la comunidad participa en la planificación y gestión de la oferta turística, algunos pobladores son asalariados y portadores de determinadas ideas turísticas expresadas en distintas iniciativas.

Hiernaux-Nicolas et al. (2002) señalan que

el impacto que el turismo pueda tener en el desarrollo nacional de los respectivos países, podría asociarse a modelos de desarrollo turístico, que evidencia la forma en como se ha desarrollado el turismo en términos económicos y sociales. Existen tres tipos; el modelo segregado, el modelo relativamente integrado y el modelo integrado, en este se supone una mayor participación de las poblaciones locales en la gestión del turismo... no solo entrarían como asalariados, sino como portadores de determinadas ideas turísticas expresadas en distintas iniciativas. En lo que respecta a la organización empresarial lo que aquí prevalecería sería la pequeña y mediana empresa.

Lidia Mendoza, encargada de promoción en la sub delegación INTUR, San Juan del Sur (comunicación personal, 4 de octubre 2017), destacó que el turismo rural comunitario se implementa en la comunidad El Ostional con el fin de brindar al turista un ambiente más relajado, convivencia con la comunidad local y ser partícipe de las actividades tradicionales como la pesca, agricultura y compartir conocimientos entre y con los pobladores, ambiente distinto a otras zonas donde se practica el turismo de forma masiva.

Nicacio (2017) señala que el turismo rural comunitario es una actividad compatible con el desarrollo sostenible debido a que ha demostrado ser una actividad socialmente responsable que no pone en riesgo los recursos y condiciones para el desarrollo de las futuras generaciones, siendo su característica principal lo vivencial, es decir, permite al turista involucrarse y participar en actividades tradicionales de la comunidad local.
José de la Cruz, presidente de COOPETUR (comunicación personal, 4 de octubre del año 2017) afirma que, el turismo rural está siendo manejado por los miembros de la comunidad, eso lo hace comunitario, nos encargamos de la planificación y gestión para desarrollar la actividad, empezamos desde el 2002 como cooperativa turística y hemos tenido muchas dificultades, porque no conocíamos muy bien esta actividad. Respecto a esto Nicacio (2017) menciona que este tipo de turismo por su naturaleza comunitaria requiere de la participación de miembros de la población de las comunidades locales en las zonas donde se desarrolla. Los emprendimientos en el sector turismo emplean una fuerza laboral conformada, en su mayoría, por pobladores locales.

Existen diferentes motivos para el origen de las iniciativas de turismo comunitario, entre ellos Cañada (2014) señala a: organizaciones comunitarias preocupadas por el medio ambiente, grupos de mujeres que tratan de incrementar sus recursos y poder ser más independientes, pequeños negocios que se asocian para potenciar y complementar su oferta, y familias y cooperativas campesinas que buscan mejorar su economía. En la comunidad El Ostional el desarrollo de esta actividad turística, amplía la visión productiva tradicional del sector pesquero y agropecuario, contribuyendo a la revalorización del territorio desde nuevas perspectivas productivas, también se desarrolla la inclusión de jóvenes, mujeres y ancianos que con su aporte al desarrollo de la actividad turística y su valor cultural hacen que el turismo rural comunitario sea atractivo a los turistas. Todo esto es parte de la neorruralidad, donde toma énfasis la multifuncionalidad de las áreas rurales. Tal es el caso de la finca Magdalena en la Isla de Ometepe, Rivas, donde el turismo ayudó a reactivar la economía e impulsar otras actividades como la producción de café (Cañada, 2014, p. 19).

Organización. El $30 \%$ de las familias mencionan que un miembro del hogar pertenece a alguna organización. En la comunidad existen varias cooperativas y organizaciones que trabajan en pro del desarrollo, entre ellas están: la COOPETUR, cooperativa agropecuaria de varones Genaro Pizarro, Cooperativa de buzos Pablo René Collado, cooperativa agropecuaria de mujeres María Luisa y cooperativa de Ostras. Según Kieffe (2018) la acción de emprender una actividad turística remite a un proceso de construcción social que usa recursos para crear dinámicas territoriales. El turismo se puede entender como una obra producida por las acciones de quienes participan en ella, implica ejercicio de reflexividad, racionalidad, intencionalidad y control por parte de los actores locales sobre sus actividades y los contextos físicos y sociales. Esto está ligado con el objetivo del Turismo Rural Comunitario señalado por Pérez et al. (2010) al indicar que: 
La comunidad rural es la que se organiza para ofrecer servicios y a la vez, controlar el ingreso que se obtiene de los mismos. Esto deja claro que la acción colectiva es la clave en esta propuesta. El rol de los líderes ya sea en organización, promoción o administración y la construcción de una institucionalidad con reglas transparentes, son elementos complementarios que pueden llevar al éxito o al fracaso el turismo rural comunitario.

En el caso de la comunidad El Ostional José de la Cruz (comunicación personal, 4 de octubre 2017), expresó que la cooperativa de turismo, tiene una junta directiva que se ha encargado de organizar, delegar el trabajo de cada uno de los miembros y de ofertar el turismo a nivel local; todo esto ayuda a comercializar sus productos y poder distribuirlo de manera equitativa. En la actualidad a variado un poco, ellos siguen trabajando de forma organizada, pero cuando la afluencia de turistas es baja, cada uno se encarga de conseguir sus propios turistas y así mantenerse en el mercado.

La organización en la planificación y desarrollo de la actividad turística rural comunitaria es de vital importancia y según el nivel organizativo en que se encuentren, permitirá el acceso o la visualización de oportunidades, en las que aprovechando sus fortalezas en términos de recursos y de atractivos turísticos podrán incursionar en un nuevo mercado o actividad. Todo esto concuerda con Cañada y Gascón (2007, p.74), quien manifiesta que el turismo rural comunitario "es un turismo de pequeño formato, establecido en zonas rurales $\mathrm{y}$ en el que la población local, a través de sus estructuras organizativas, ejerce un papel significativo en su control y gestión". Flores et al. (2014) mencionan que, en el turismo rural y comunitario, la población local es parte esencial para permitir un proceso de desarrollo competitivo y cuidadoso con el ambiente, además propicia un equilibrio en lo referente a sus valores éticos, morales, sociales y culturales, integra y fortalece la economía comunitaria, mejora las condiciones de vida y de trabajo de sus miembros.

Equidad de género y función de la mujer. En la actualidad, la junta directiva de COOPETUR, está conformada por 80 $\%$ de mujeres. Estas mujeres juegan un papel importante opinando y participando en todas las decisiones relacionadas con la actividad turística. En general el $57 \%$ de las mujeres se sienten escuchadas, son ellas las que se encargan de sus negocios y son parte fundamental de la gestión y el desarrollo turístico. Pérez et al. (2010) mencionan que:

Los liderazgos están asociados a las formas de organización de las iniciativas. En el caso de las cooperativas agrarias, el liderazgo tiende a ser masculino y ligado a las características de la producción agraria. En el turismo las mujeres han ido ganando espacios a nivel de los roles y de las juntas directivas locales, se les asocia a la organización y a la distribución de los roles de cocina, limpieza, atención a turistas y, principalmente, en la administración de los ingresos generados por el Turismo Rural Comunitario. Algunos ejemplos que mencionan es la Cooperativa de Turismo Rural Comunitario Las Pilas-El Hoyo, en el que las mujeres llevan el manejo de las finanzas y en la casa comunal La Granadilla las mujeres se encargan de la atención a turistas, servicios de alimentación y manejo de las finanzas.

Para Pérez y Fuller (2015) la mujer comienza a ejercer un renovado papel en los procesos de desarrollo de los territorios rurales asumiendo nuevas funciones, y es en la actividad turística donde esas funciones adquieren especial importancia porque les permite incorporarse al mercado del trabajo local, ya sea como empresarias, creando y gestionado sus propios negocios o como trabajadoras por cuenta ajena.

El Ostional no está exento de esta característica ya que según José de la Cruz (comunicación personal, 4 de octubre 2017) de los 14 miembros de COOPETUR, algunos son propietarios de alojamientos y comedores, de estos establecimientos la mayoría son administrados por mujeres; esto ha hecho que la mujer sea reconocida en la comunidad por su participación y aporte a la economía de su hogar. Estas mujeres desempeñan cargos de administradoras de sus propios negocios, además que son las que acceden a financiamientos otorgados por INTUR y el programa de microcrédito Usura Cero (programa de gobierno). También son favorecidas con capacitaciones y otros beneficios de organismos privados como Grupo Pellas y Paso Pacífica.

Rico y Gómez (2009, p. 53) consideran a la mujer rural y a su capacidad laboral como elementos imprescindibles de cara al inicio de nuevas actividades económicas dentro del proceso de diversificación productiva de las zonas rurales, aumentando así las tasas de actividad, generando nuevos empleos $\mathrm{y}$, en general, impulsando de forma efectiva las nuevas estrategias en el ámbito rural. Un ejemplo a nivel de Latinoamérica según Pérez y Fuller (2015) es el de un grupo de campesinos e indígenas que constituyeron en el 2006 una red de turismo rural comunitario, para ofertar productos y servicios turísticos, estas iniciativas, conformadas mayoritariamente por mujeres, constituyen una base de análisis de estos nuevos tipos de turismo rural, basados en la participación local y en la mejora de la condición de las mujeres que permitió la posibilidad de autonomía personal y empoderamiento de género.

Capacitaciones. El $59 \%$ de los hogares tienen al menos una persona capacitada, estas capacitaciones han sido brindadas por organismos nacionales e internacionales, entre ellos el INTUR, Grupo Pellas con el proyecto Triangulo del Sur, Paso Pacífica y el Instituto Nacional de Aprendizaje de Costa Rica (INA-CR). 
Según Yader Collado, representante de turismo de la Alcaldía de San Juan del Sur y Lidia Mendoza, encargada de promoción del INTUR-San Juan del Sur (comunicación personal, 4 de octubre del año 2017), se han desarrollado capacitaciones en temas relacionados con el turismo, entre ellas, bartender, camarería, cocina, atención al cliente, guarda parques, manipulación de alimentos y han realizados planes de negocios y cursos básicos de administración.

Estas capacitaciones se han impulsado desde COOPETUR y han motivado a los pobladores de El Ostional a realizar acciones de emprendimiento, así como continuar con estudios de secundaria y en la educación superior. Según la Organización Mundial del Turismo (OMT, 1998) la formación de calidad es indispensable para obtener profesionales capaces de satisfacer las necesidades de los clientes, motivados e involucrados en la organización en la que trabajan; según Pérez et al. (2010) en Guatemala y Nicaragua el liderazgo comunitario, tiende a ser un elemento clave en la asignación de roles, en la asignación de ingresos individuales y en la inversión comunitaria.

Según Pérez et al. (2010) la incorporación de actividades turísticas en las zonas rurales ha impulsado la creación de diversos tipos de empleo y paralelamente, los miembros de las comunidades han accedido a participar en capacitaciones pertinentes para la implementación del turismo como estrategia en el modelo de desarrollo turístico rural comunitario, debido a que se necesita tener conocimientos acerca del tema y de esta forma, se tengan las herramientas necesarias para poder desarrollar una buena planificación y gestión de calidad en esta actividad.

Szmulewicz (2000), cita a Solsona (1997, p. 20), quien menciona que "la educación, la formación ocupacional y la formación profesional, así como la sensibilización social y la motivación de los recursos humanos y el fomento de la cultura local son medidas estratégicas para el futuro de las áreas rurales" así mismo Szmulewicz (2000), comenta que las empresas de turismo rural, participan en acciones de capacitación por la necesidad de mejorar la cualificación de sus trabajadores, para adaptarse a los cambios tecnológicos y adecuarse a los cambios en las demandas turísticas de los consumidores (aumentar la calidad y la satisfacción de los clientes).

Percepción de mejoría en la economía comunitaria. El $42.6 \%$ de los pobladores perciben que la economía familiar ha mejorado, mientras que un $36.1 \%$ consideran que no ha habido mejora económica. Esto tiene un fuerte vínculo con los objetivos a los que apunta el turismo rural. Pérez (2010, p.512) nos dice que esta actividad debe partir de "la integración con el resto de las actividades económicas, dando lugar a la diversificación productiva del área rural propiciando las iniciativas tendentes a mejorar la presentación del patrimonio inmobiliario, reactivar el comercio, promover el sector artesanal, entre otras".
Según Julio Lacayo, Secretario Político de El Ostional (comunicación personal, 4 de octubre 2017), muchos pobladores han invertido en la mejora de sus hogares y establecimientos, ese es un indicio de mejora económica.

José de la Cruz (comunicación personal, 4 de octubre 2017), concuerda que las casas han mejorado en su fachada y que los establecimientos turísticos se han ampliado, comenzaron con una o dos habitaciones y ahora hay más de cuatro habitaciones en los alojamientos.

Ley $\mathrm{N}^{\circ} 835$, (2013, p. 6), plantea en su artículo 8, que el Turismo Comunitario "es un modelo de gestión que promueve experiencias turísticas planificadas e integradas sosteniblemente al medio rural y es desarrollada por las poblaciones locales organizadas, para beneficio de la comunidad". La idea de realizar turismo en El Ostional nace de un grupo de pobladores que la identifican como una oportunidad de desarrollo económico, protección y para dar realce a sus actividades culturales, espacios rurales y sus atractivos naturales. Casas-Jurado et al. (2012) concuerda con que el objetivo principal del turismo comunitario es mejorar el nivel de vida de los habitantes, garantizando la preservación de su identidad étnica y para lograrlo, el turismo comunitario debe ser una actividad complementaria, no sustituta de las actividades agrícolas tradicionales.

Según Pérez et al. (2010) en las experiencias nicaragüenses, el Servicio Holandés de Cooperación al Desarrollo (SNV) ha identificado la existencia de impactos positivos y negativos en las comunidades que se involucran en la actividad turística. Existen casos en los que las comunidades han abandonado la actividad productiva, y otros en los que el turismo se ha convertido para ellas en una opción complementaria en la búsqueda de una mejora en su propio nivel de vida. Los estudios han identificado cuatro ejes o indicadores de impacto: la estructura mujeres-hombres, la familia, la comunidad y el medioambiente.

\section{Vinculación del turismo con otras fuentes de ingreso}

Actividades asalariadas. El $37 \%$ de las actividades económicas asalariadas mejoran con el turismo, pero en un $63 \%$ no mejoran, si hay turismo o no para estas actividades, no reflejan un gran cambio. Se considera que este porcentaje es alto debido a que las actividades asalariadas son menos sensibles a cambios a corto plazo en el mercado laboral. Se debe tomar en cuenta que las actividades asalariadas tienen una remuneración fija y esta no aumenta por la cantidad de trabajo asignado, pero si puede generarse un nuevo puesto, debido a la demanda. De forma individual la persona no siente una mejora, pero de forma colectiva existen más fuentes de trabajo.

El turismo repercute en la economía de las localidades donde se desarrolla, aunque no en todos ellos con la misma intensidad. De acuerdo con esto Sánchez 
(2014), menciona que uno de los beneficios del turismo es la capacidad de generar riqueza y empleo, así como también puede ser un instrumento para combatir la pobreza, revitalizar e impulsar distintas zonas o territorio. Pérez (2010) concuerda diciendo que el turismo puede ser el incentivo para el desarrollo local de un gran número de zonas rurales, permite dinamizar las actividades económicas tradicionales y valorizar las particularidades culturales, ofreciendo posibilidades de empleo a jóvenes de zonas rurales.

Actividades por cuenta propia. El 61 $\%$ de la población de la comunidad se dedican a actividades por cuenta propia, entre estas la pesca, la agricultura, el turismo, el comercio entre otras. El promedio de ingresos que obtienen mensualmente es de USD 348.15. El promedio de gastos en los hogares es de USD 238.18. La diversificación de la economía es un pilar fundamental en el desarrollo de la región, se considera que las actividades por cuenta propia son un indicador del sostenido crecimiento económico de la zona.

Sánchez (2014) señala que los gastos de los turistas no sólo se limitan al pago de alquiler de una habitación, sino que también destinan parte de dinero a una variedad de servicios tales como alimentos, transportes, entretenimientos, excursiones y actividades diversas. Al beneficiar al resto de sectores de la economía se vuelve un efecto multiplicador.

Con las actividades por cuenta propia el $45 \%$ no presenta mejoría, mientras que la percepción de mejoría económica con el aumento de las llegadas de turistas es de $55 \%$, este indicador es mucho más sensible en este tipo de actividades debido a que por su naturaleza son más susceptibles a los cambios en el mercado. Por esta razón el vínculo entre actividades económicas y el turismo se estudió únicamente a partir de las actividades por cuenta propia y la percepción de mejora con la llegada de turistas.

En la Figura 1 se muestra que las actividades relacionadas con el turismo son las que perciben la mayoría de la mejora con la llegada de turistas como el turismo (hospedaje, bares, comedores, guías turísticos), seguidas por el comercio, la pesca y otras actividades como carpintería, soldadura, taller de mecánica, albañilería y costura. La agricultura no percibe ninguna mejora y se detectó que, en esta comunidad, es básicamente de subsistencia y no está involucrada directamente con el mercado.

Kieffe (2018) menciona que la introducción del turismo en las zonas rurales busca diversificar y no especializar. En este contexto el turismo rural comunitario, se puede presentar como una propuesta de diversificación de los ingresos de las familias campesinas, como una actividad posible entre otras que, sin reemplazar las actividades preexistentes, las complementaría.

Ingresos y turismo. Los pobladores manifestaron que en promedio mensual perciben ganancias de USD 373.85 por efecto directo del turismo, mientras que, en otros pobladores no ligados al turismo, su ingreso promedio mensual es de USD 313.03. Las personas que reconocieron que su actividad económica está relacionada con el turismo, se obtienen más ingresos en sus negocios que los que dicen que su actividad no está relacionada.

El turista al movilizarse a un lugar fuera de su entorno habitual demandará alimentos y bebidas, alojamiento, guías turísticos y recreación, esto se enlaza con el incremento de ingresos económicos, porque hay más gente que demanda el servicio prestado por estos negocios.

Según Pérez et al. (2010) el turismo puede ser el incentivo para el desarrollo local de un gran número de zonas rurales. Este sector en auge permite dinamizar las actividades económicas tradicionales y valorizar las particularidades culturales locales, ofreciendo posibilidades de empleo a jóvenes de zonas rurales y frenando con ello el éxodo rural.

\section{CONCLUSIONES}

El modelo integrado que se ha desarrollado en la comunidad El Ostional genera beneficios sociales y económicos a todos los integrantes de la cooperativa que de manera indirecta generan beneficios al resto de la comunidad, contribuyendo en el desarrollo de la pesca y la agricultura.

La capacidad organizativa de los habitantes de El Ostional, propicia el desarrollo de la actividad turística, 
permitiendo que la comunidad auto gestione sus recursos, de forma sostenible y planificada. Una de las máximas expresiones de la capacidad organizativa, es la conformación de cooperativas de pesca, agricultura y turismo.

En el modelo de desarrollo turístico rural comunitario que se gestiona en la comunidad El Ostional, se identifica incremento económico en los hogares relacionados directa o indirectamente a la actividad turística y mejora en la infraestructura básica como escuelas, caminos y puestos de salud.

El turismo es una actividad complementaria en la economía tradicional de la comunidad y ha resultado ser una alternativa para superar las dificultades sociales y económicas.

\section{AGRADECIMIENTOS}

A las autoridades locales y pobladores de la comunidad El Ostional, por abrir las puertas de sus hogares y brindar información, para poder tener una prueba fehaciente del aporte de la actividad turística en la comunidad. Al MSc. Roberto Carlos Larios González por su apoyo y constante motivación en la redacción de este artículo, sin su ayuda esto no hubiese sido posible.

\section{REFERENCIAS BIBLIOGRÁFICAS}

Asamblea Nacional de Nicaragua. (2013, 8 de marzo). Ley No 835. Ley de Turismo Rural Sostenible de la República de Nicaragua. Diario Oficial No. 184. https://rb.gy/bvjhfl

Cañada, E. y Gascon, J. (2007). Turismo y desarrollo: herramientas para una mirada crítica. Enlace. https://rb.gy/il1 kgc

Cañada, E. (2014). Turismo comuitario en Centroamérica, experiencias y aprendizajes. Enlace. http://www.albasud.org/publ/docs/69.pdf

Casas-Jurado, A., Soler Domingo, A. y Jaime Pastor, V. (2012). El turismo comunitario como instrumento de erradicación de la pobreza: potencialidades para su desarrollo en Cuzco (Perú). Cuaderno de turismo, (30), 91-108. https://revistas.um.es/turismo/article/ view/160461

Banco Central de Nicaragua. (2018). Cuenta satelite de turismo de Nicaragua 2017. https://www.bcn.gob.ni/publicaciones/periodicidad/ anual/satelite turismo/2017/CSTN.pdf

Flores Amador, C., Zizumbo Villarreal, L., Cruz Jiménez, G. y Vargas Martínez, E. (2014). Economía social, comunalidad: orientación teórica para el turismo rural, como alternativa de desarrollo. Revista mexicana de ciencias agrícolas, 5(9), 1645-1658. https://doi. org/10.29312/remexca.v0i9.1054

Hiernaux-Nicolas, D., Cordero, A. y Van, L. (2002). Imaginarios sociales y turismo sostenible. FLACSO. http://biblioteca.clacso.edu. ar/ar/libros/costar/cua123.pdf

Kieffe, M. (2018). Conceptos claves para el estudio del Turismo Rural Comunitario. El periplo sustentable, (34), 8-43. http://www.scielo. org.mx/scielo.php?script=sci_arttext\&pid=S1870-90362018000100008

Nicacio, C. (2017). Turismo Rural Comunitario: Una tentadora alternativa para futuros emprendedores. InnovaG, (2), 49-55. http:// revistas.pucp.edu.pe/index.php/innovag/article/view/18748/18988

Organización Mundial del Turismo. (1998). Introduccion al turismo. http://www.utntyh.com/wp-content/uploads/2011/09/ INTRODUCCION-AL-TURISMO-OMT.pdf

Pérez, F., Barrera, O., Peláez, A. y Lorío, G. (2010). Turismo Rural Comunitario, como Alternativa de Reducción de la Pobreza Rural en Centroamérica. Nitlapan -UCA. http://repositorio.uca.edu.ni/236/1/cuaderno 36.pdf

Pérez Galán, B. y Fuller, N. (2015). Turismo rural comunitario, género y desarrollo en comunidades campesinas e indígenas del sur del Perú. Quaderns, (31), 95-119. https://www.raco.cat/index.php/QuadernsICA/article/view/308759

Pérez, S. (2010). El valor estratégico del turismo rural como alternativa sostenible de desarrollo territorial rural. Agronomía Colombiana, 28(3), 507-513. https://www.redalyc.org/articulo.oa?id=180320698018

Rico, M. y Gómez, J. (2009). La contribución de la mujer en la economía rural de Castilla y León. Economía Agraria y Recursos Naturales, 9(2), 51-77. https://dialnet.unirioja.es/servlet/articulo?codigo=3115714

Sánchez Rivera, A. (2014). El turismo rural y su incidencia en el desarrollo socioeconómico de un territorio: en el caso de la comarca de la Sidra [Tesis de maestría, Universidad de Oviedo]. Repositorio Institucional de la Universidad de Oviedo. https://digibuo.uniovi. es/dspace/handle/10651/27986

Szmulewicz, P. (2000). Calidad de recursos humanos para un turismo rural sustentable. Gestión turística, (5), 19-30. https://doi. org/10.4206/gest.tur.2000.n5-03 